\title{
ARTICLE
}

Multiple myeloma gammopathies

\section{Detection of MYD88 and CXCR4 mutations in cell-free DNA of patients with IgM monoclonal gammopathies}

\author{
Tina Bagratuni ${ }^{1}$ - loannis Ntanasis-Stathopoulos $\mathbb{D}^{1} \cdot$ Maria Gavriatopoulou $^{1}$. Nefeli Mavrianou-Koutsoukou ${ }^{1}$. \\ Christine Liacos $^{1}$ - Dimitrios Patseas ${ }^{1}$ - Nikolaos Kanellias ${ }^{1} \cdot$ Magdalini Migkou ${ }^{1}$ - Dimitrios C. Ziogas ${ }^{1}$. \\ Evangelos Eleutherakis-Papaiakovou ${ }^{1} \cdot$ Maria Roussou $^{1} \cdot$ Despina Fotiou $^{1} \cdot$ Evangelos Terpos $\mathbb{D}^{1} \cdot$ \\ Efstathios Kastritis $\mathbb{D}^{1} \cdot$ Meletios A. Dimopoulos ${ }^{1}$
}

Received: 19 January 2018 / Revised: 29 May 2018 / Accepted: 7 June 2018 / Published online: 19 July 2018

(c) The Author(s) 2018. This article is published with open access

\begin{abstract}
Liquid biopsyis being integrated into cancer diagnostics with profound therapeutic implications. However, its role in Waldenström's Macroglobulinemia (WM) and IgM monoclonal gammopathies is still unclear. In this study, we evaluated the role of peripheral blood (PB) cell-free DNA (cfDNA) in characterizing the mutational status of MYD88 and CXCR4 of patients with IgM monoclonal gammopathies. Paired bone marrow (BM) tumor DNA (tDNA) and PB cfDNA samples from 98 patients (9 MGUS, 45 with WM in remission, 44 with smoldering WM, newly diagnosed or relapsed WM) and 10 controls with non-IgM monoclonal gammopathies were analyzed. Regarding MYD88 ${ }^{\mathrm{L} 265 \mathrm{P}}$ mutation, 76 patients had paired tDNA and cfDNA informative samples. Among patients with WM in remission, 65\% harbored the MYD88 ${ }^{\mathrm{L} 265 \mathrm{P}}$ mutation, whereas the corresponding percentage among smoldering/newly diagnosed or relapsed WM was $92 \%$. The overall concordance rate was 94\% (72/76). For CXCR4 mutations, 65 patients had paired informative tDNA and cfDNA samples. The overall concordance rate was 90\% (59/65). All controls had wild-type MYD88 and CXCR4. In conclusion, PB cfDNA is a useful, minimally invasive, cost-effective, and time-effective tool for the identification of the presence of MYD88 and CXCR4 mutations in patients with IgM monoclonal gammopathies avoiding unnecessary BM assessment.
\end{abstract}

\section{Introduction}

Whole-genome sequencing has identified highly recurring somatic mutations in patients with Waldenström's Macroglobulinemia (WM) and IgM Monoclonal Gammopathy of Undetermined Significance (MGUS) [1, 2]: 76-100\% of WM patients and 43-87\% of those with IgM MGUS harbor a single point mutation in MYD88 gene (rs387907272), resulting in p.Leu265Pro (L265P) amino acid change [1-5]. MYD88 is an adapter for Toll-like and interleukin-1 (IL1) receptors, and the MYD $88^{L 265 P}$ mutation results in

These authors contributed equally: Tina Bagratuni, Ioannis NtanasisStathopoulos.

Meletios A. Dimopoulos

mdimop@med.uoa.gr

1 Department of Clinical Therapeutics, School of Medicine, National and Kapodistrian University of Athens, Athens, Greece constitutive activation of nuclear factor $\mathrm{\kappa B}$ through IL1 receptor-associated kinase and Bruton's tyrosine kinase (BTK) [1, 6-8]. The presence of MYD88 ${ }^{L 265 P}$ mutation provides a significant evidence for the diagnosis of WM but has also been associated with improved probability and quality of clinical responses to the BTK inhibitor ibrutinib [9-11]. Recurrent activating somatic mutations (frameshift or nonsense) are also found in $\mathrm{C}-\mathrm{X}-\mathrm{C}$ chemokine receptor type 4 (CXCR4) gene in a frequency of about $30-40 \%$ of patients with WM and less often in those with IgM MGUS, representing the second most mutated gene in WM $[2,3,12]$. CXCR4 is a G-protein-coupled receptor, acting as a key regulator of cell trafficking in heamatopoietic stem cells and clonal B cells, also interacting with the related ligand stromal-derived factor 1 [13-16]. Studies have shown the relevance of specific CXCR4 mutations to enhanced WM cell dissemination leading to in vivo disease progression as well as increased resistance to ibrutinib treatment [17-19]. Both MYD88 and CXCR4 mutations are detected in the bone marrow (BM), although there have been attempts to detect them in $\mathrm{CD} 19^{+}$-selected cells in the 
peripheral blood $(\mathrm{PB})$ of patients with $\operatorname{IgM}$ monoclonal gammopathies [20, 21].

Analysis of tumor-derived circulating nucleic acids, such as circulating cell-free tumor DNA (cfDNA) (termed also as liquid biopsy) is currently being integrated in diagnosis, prognosis, disease monitoring, and early detection of clonal evolution of several different solid tumors [22]. cfDNA may reflect the genomic alterations within the whole tumor compartment and may also substitute the need for invasive tissue sampling in specific situations [23]. In hematological malignancies, cfDNA may be used as a surrogate marker of response to treatment, minimal residual disease (MRD), and early detection of clonal evolution and relapse in patients with B-cell Non-Hodgkin Lymphoma and Chronic Lymphocytic Leukemia [24-26]. There is also accumulating evidence indicating the role of cfDNA in patients with multiple myeloma (MM) as a universal marker of tumor burden with prognostic implications [27, 28].

The aim of our study was to investigate the role of cfDNA in the characterization of the mutational status of MYD88 and CXCR4 of patients with IgM monoclonal gammopathies.

\section{Materials and methods}

PB (10-12 mL) and BM aspirates $(5-10 \mathrm{~mL})$ were collected from 98 patients, including 24 patients with smoldering WM, 11 with symptomatic WM, 9 with IgM MGUS, as well as 10 control donors with non-IgM monoclonal gammopathies; their characteristics are summarized in Table 1.

Table 1 Patient characteristics

\begin{tabular}{ll}
\hline IgM patients $(n)$ & 98 \\
IgM MGUS & 9 \\
Asymptomatic WM & 23 \\
Newly diagnosed symptomatic WM & 12 \\
WM in relapse & 9 \\
WM during Tx and in remission & 17 \\
WM post Tx & 28 \\
Kappa/Lamda & $79 / 19$ \\
Age (median, range in years) & $72.5(23-88)$ \\
Bone marrow infiltration $(\% ;$ median, range) & $25(0-90)$ \\
IgM levels (median, range in mg/dL) & $1260(85-7810)$ \\
Controls $(n)$ & 10 \\
Symptomatic MM $(n)$ & 5 \\
Smoldering MM $(n)$ & 2 \\
Non-IgM MGUS $(n)$ & 3 \\
Age (years; median, range) & $67(57-81)$ \\
\hline
\end{tabular}

IgM immunoglobulin M, WM Waldenström's Macroglobulinemia, $M M$ multiple myeloma, MGUS monoclonal gammopathy of undetermined significance, $L C$ light chain
The BM infiltration in patients with smoldering WM was $30 \%$ (range $12-80 \%$ ), newly diagnosed symptomatic WM was $60 \%$ (range 14-90\%), and in WM in relapse was 38\% (range 20-85\%). PB was collected in EDTA tubes and processed immediately for DNA extraction using the MagMax cell-free DNA isolation kit (Thermo Fisher Scientific), according to manufacturer's instructions. The quantity of cfDNA was measured by qubit fluorometer using the HS dsDNA kit (Thermo Fisher Scientific). cfDNA concentration varied between 6 and $80 \mathrm{ng} / \mu \mathrm{L}$. BM aspirates were collected at the same time with $\mathrm{PB}$, and were processed for CD19 enrichment [1, 29]. Briefly, mononuclear cells from BM aspirates were isolated by Ficoll-Paque gradient centrifugation followed by positive selection using CD19 magnetic beads BM (Miltenyi Biotech). DNA of CD19 ${ }^{+}$-selected cells (tDNA) was extracted using Allprep DNA/RNA mini kit (QIAGEN, Valencia, CA) and quantified using the HS dsDNA kit. The paired tDNA and cfDNA samples from patients and controls were analyzed for the mutation detection of $M Y D 88^{L 265 P}$ in the exon 5 of MYD88 gene and for $C X C R 4$ gene in all exons.

The detection of $L 265 P$ mutation by allele-specific PCR (AS-PCR) and direct sequencing in both tDNA and cfDNA was performed as previously described [30]. Specifically PCR was performed with up to $15 \mathrm{ng}$ of cfDNA or tDNA (depending on DNA availability in each case). Thermal cycling conditions were as follows: five minutes at $94^{\circ} \mathrm{C}$ followed by 35 cycles of amplification using $60 \mathrm{~s}$ at $94^{\circ} \mathrm{C}$, $60 \mathrm{~s}$ at $60^{\circ} \mathrm{C}$, and $60 \mathrm{~s}$ at $72^{\circ} \mathrm{C}$, with subsequent $5 \mathrm{~min}$ extension at $72^{\circ} \mathrm{C}$. AS-PCR was performed with specific forward primers with a single base substitution at the end of the primer: MYDW-F, 5'-GTGCCCATCAGAAGCGCCT $-3^{\prime}$ (wild type), MYDM-F, 5'-GTGCCCATCAGAAGCG CCC-3' (mutant), and MYD-R, 5'-GACGTGTCTGT GAAGTTGGCATCTC-3' (reverse). MYD88 wild-type and MYD88 mutant amplified 224 base pair (bp) products and were visualized in $1.5 \% \mathrm{EtBr}$ agarose gel. The PCR conditions for MYD88 used for direct sequencing were the same as above. The reverse primer was the same as for the AS-PCR, while the forward sequence for sequencing was MYD_F, 5'-GGGATATGCTGAACTAAGTTGCCAC-3' (forward). The amplification of the above PCR resulted in a 726 bp product. Sequencing of CXCR4 was performed using the following primers: CXCR4_1, 5'-GCTGAA TTGGAAGTGAATGTCC-3' (forward), CXCR4_1, 5'-GT CATCTACACAGTCAACCTCT-3' (reverse), CXCR4_2, 5'-CCGTGGCAAACTGGTACTTT-3' (forward), CXCR 4_2, 5'-TCACTCCAGCTAACACAGATG-3' (reverse). Amplicon sizes for CXCR4 1 and 2 were 389 and $775 \mathrm{bp}$, respectively. As cfDNA size is small ( 166 bp), we designed primers for CXCR4 gene targeting smaller amplicon sizes (100-220 bp) and amplified cfDNA samples 
Table 2 CXCR4 primers

\begin{tabular}{|c|c|c|c|}
\hline Name & Primer & Direction & Amplicon size (bp) \\
\hline \multirow[t]{2}{*}{ CXCR4_1 } & 5'-ACTGGGTTAATGCTTGCTGA-3' & $\mathrm{F}$ & 184 \\
\hline & 5'-TGCCCACCATCTACTCCATC-3' & $\mathrm{R}$ & \\
\hline \multirow[t]{2}{*}{ CXCR4_2 } & 5'- GCCCACCATCTACTCCATCA-3' & $\mathrm{F}$ & 158 \\
\hline & 5'-TGTCATCACGCTTCCCTTCT-3' & $\mathrm{R}$ & \\
\hline \multirow[t]{2}{*}{ CXCR4_3 } & 5'-TGTCATCACGCTTCCCTTCT-3' & $\mathrm{F}$ & 219 \\
\hline & 5'-AAGGTGGTCTATGTTGGCG-3' & $\mathrm{R}$ & \\
\hline \multirow[t]{2}{*}{ CXCR4_4 } & 5'-AAGGTGGTCTATGTTGGCGT-3' & $\mathrm{F}$ & 162 \\
\hline & 5'-TTCAGCACATCATGGTTGGC-3' & $\mathrm{R}$ & \\
\hline \multirow[t]{2}{*}{ CXCR4_5 } & 5'-ATCTGTGACCGCTTCTACCC-3' & $\mathrm{F}$ & 216 \\
\hline & 5'-CCTGTTGGCTGCCTTACTAC-3' & $\mathrm{R}$ & \\
\hline \multirow[t]{2}{*}{ CXCR4_6 } & 5'-CTCATCCTGGCTTTCTTCGC-3' & $\mathrm{F}$ & 173 \\
\hline & 5'-TGTTGTCTGAACCCCATCCT-3' & $\mathrm{R}$ & \\
\hline \multirow[t]{2}{*}{ CXCR4_7 } & 5'-CATCCTCTATGCTTTCCTTGGAG-3' & $\mathrm{F}$ & 171 \\
\hline & 5'-TCACTCCAGCTAACACAGATG-3' & $\mathrm{R}$ & \\
\hline
\end{tabular}

that were largely degraded. The primers used are shown in Table 2.

The thermal conditions for the above reactions were the same as in MYD88. Amplification and sequencing of MYD88 and CXCR4 for each sample was performed once, unless unsuccessful result required the repetition of either method. The study was approved by the institutional review board and all patients provided written informed consent for sample collection and analysis.

\section{Results}

\section{MYD88 genotype assessment in cfDNA and tDNA}

Of the 98 patients with IgM monoclonal gammopathies, the mutational status of MYD88 could be evaluated in 84 patients $(84 / 98$ patients, $86 \%$ ) by tDNA and in 84 patients (84/98 patients, $86 \%$ ) by cfDNA. In 14 patients tDNA (14/ $98,14 \%)$ and in 14 patients cfDNA (14/98, 14\%), MYD88 status could not be assessed due to inability of BM aspiration or to low DNA quantity, respectively. Most patients with insufficient tDNA had very low BM infiltration by lymphoplasmacytic cells: three patients had IgM MGUS, four had smoldering WM (sWM), one was newly diagnosed WM, and six had WM in remission after therapy; however, in $57 \%$ of these patients (8/14) cfDNA was informative. Among the 14 patients in which cfDNA could not be obtained, 2 had IgM MGUS, 3 were sWM, 1 was newly diagnosed (ND)WM with relatively low BM infiltration $(\sim 12 \%)$, and 8 had WM in remission.

We next sought to clarify the feasibility of using cfDNA for the detection of $M Y D 88^{L 265 P}$ mutation in 84 patients where cfDNA was available. The mutation was detected in the cfDNA of 68 out $84(81 \%)$ patients. As shown in Fig. 1, the somatic mutation L265P in the MYD88 gene is clearly detectable in cfDNA samples by AS-PCR agarose gel electrophoresis. To verify the validity of the results seen by AS-PCR in the agarose gel electrophoresis, the mutated product was also analyzed by direct sequencing. The MYD88 mutational analysis was also confirmed by direct sequencing, where the quality of the images is also shown in Fig. 1. The feasibility of using cfDNA for determining the MYD88 mutation status is equally informative to that determined by tDNA (Fig. 1). In samples with MYD88 ${ }^{\mathrm{WT}}$, only the wild-type amplicon product was observed (Fig. 1).

To determine the sensitivity of the AS-PCR assay for the cfDNA samples we performed serial dilutions of a mutated cfDNA product. The sensitivity assessment of AS-PCR in cfDNA demonstrated that $M Y D 88^{L 265 P}$ mutation was detectable in sample product with a low DNA concentration $(5 \mathrm{ng}$ ) in a dilution of up to $1 \%$ (as low as $0.05 \mathrm{ng}$ of DNA), where the mutated band is clearly detectable (Fig. 2). Our results show that further dilutions are possible. In parallel we examined the use of both conventional AS-PCR and direct sequencing for the detection of the MYD $88^{L 265 P}$ mutation in tDNA derived from CD19-selected BM cells. The mutation was detected in 71 out of 84 patients $(84 \%)$. All patients in both cfDNA and tDNA, positive or negative for L265P MYD88 by AS-PCR, were also positive or negative by direct sequencing, respectively. The results showed complete reproducibility among replicates between different runs.

Among tested patients, 76 patients had both tDNA and cfDNA informative samples. MYD $88^{L 265 P}$ mutation was detected in 62 out of 76 patients $(82 \%)$ in both tDNA and cfDNA; in 4 out of $76(5 \%)$ the mutation was seen in tDNA but not in cfDNA and 10 out of $76(13 \%)$ patients harbored the $M Y D 88^{W T}$ genotype both in tDNA and cfDNA. Thus, the 


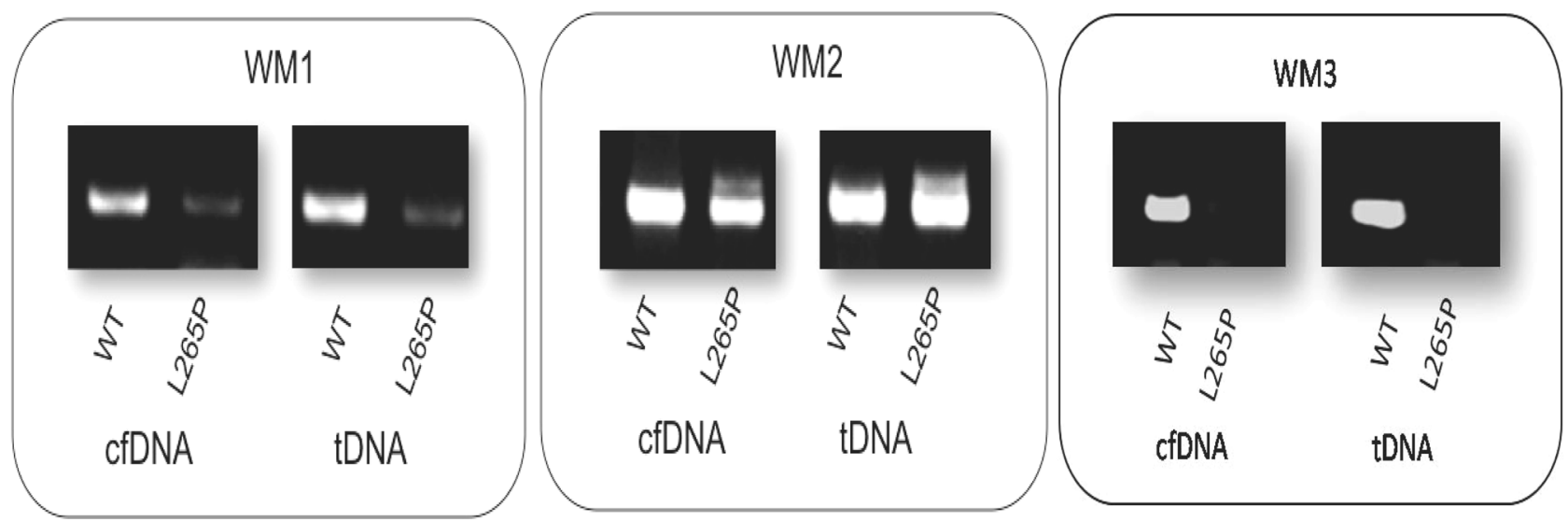

WM1 cfDNA

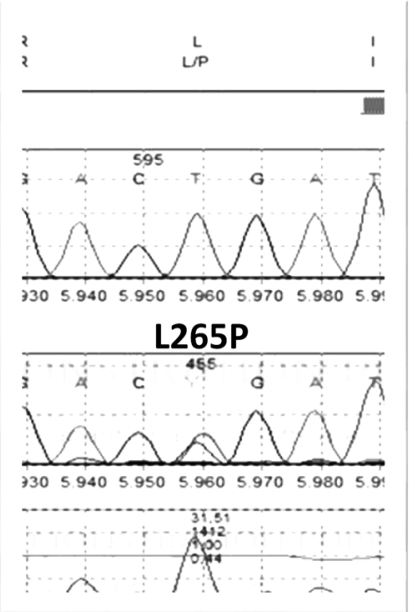

WM3 cfDNA

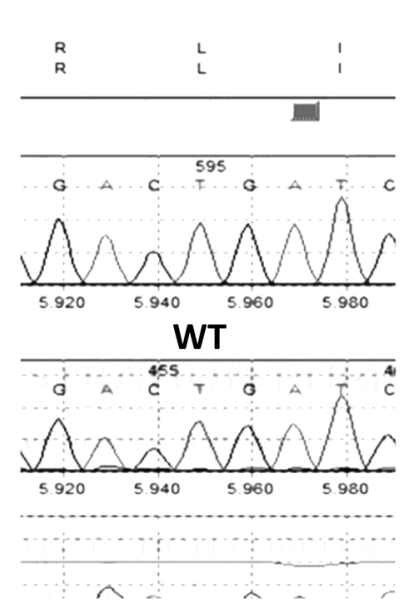

Fig. 1 AS-PCR and Sanger sequencing for MYD88 L265P in tDNA and cfDNA. WM1 and WM2 present in both wild-type and mutant products, while WM displays oonly the wild-type allele. WM1 and WM3 cfDNA sequencing analysis is also displayed

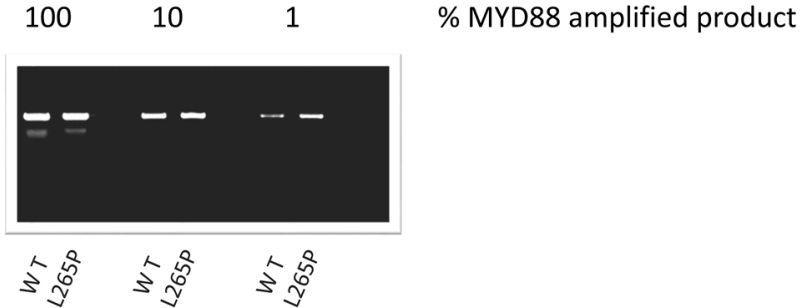

Fig. 2 The sensitivity assessment of AS-PCR in cfDNA MYD88 wildtype and mutated product as seen on a $1.5 \%$ agarose-gel electrophoresis. Both the wild-type and the mutant alleles were detected to a dilution of $1 \%$

overall concordance between tDNA and cfDNA for MYD88 genotype was $94 \%$ ( 72 out of 76 patients). Among patients with IgM MGUS, WM in remission, and sWM/NDWM/ relapsed (RR) WM, the concordance rates were $100 \%$ (6 out of 6 patients), 97\% (33 out 34 patients), and 91\% (33 out of 36 patients), respectively. The frequency of detection of
MYD $88^{L 265 P}$ in tDNA was very similar for patients with sWM (95\% MYD88 ${ }^{L 265 P}$ ) vs. NDWM $(91 \%)$ vs. WM in relapse

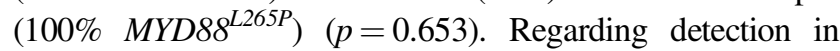
cfDNA was also similar for patients with sWM $(82 \%$ $\left.M Y D 88^{L 265 P}\right)$ vs. NDWM (73\%) vs. WM in relapse $(100 \%$ $\left.M Y D 88^{L 265 P}\right)(p=0.439)$. Overall, the MYD $88^{L 265 P}$ mutation by either cfDNA or tDNA was present in 6 out of 6 MGUS patients (100\%), 27 out of 34 patients with disease in remission (79\%), and in 36 out of 44 patients with smoldering or newly diagnosed or relapsed disease $(81 \%)$.

Regarding the control group of non-IgM MGUS and MM patients, there were no MYD88 mutations detected in tDNA ( 8 out of 8 for MYD88 and 8 out of 8 patients for CXCR4) and cfDNA (6 out of 6 patients for MYD 88 and 7 out of 7 patients for $C X C R 4)$. One patient with marginal zone lymphoma harbored the MYD $88^{L 265 P}$ mutation detected both in tDNA and cfDNA.

We also evaluated whether the degree of BM infiltration, which grossly reflects the burden of the clone and IgM 
levels were associated with mutation detection rates. In univariate analysis ( $t$-test, two-sided), the probability of identifying MYD88 ${ }^{\mathrm{L} 265 \mathrm{P}}$ in $\mathrm{CD}^{+} 9^{+}$cells was marginally associated with the BM infiltration $(p=0.062)$ and was associated with $\operatorname{IgM}$ levels $(p=0.012)$. Regarding MYD88 $^{\text {L265P }}$ detection by cfDNA this was not significantly associated with BM infiltration $(p=0.126)$ and was associated with IgM levels $(p=0.045)$. The amount of cfDNA was marginally associated with BM infiltration $(p=0.083)$ and not with IgM levels ( $p=0.188)$; on the contrary, and as expected, there was an association of collected CD19 ${ }^{+}$cells with BM infiltration $(p=0.012)$, but not with IgM levels $(p=0.074)$. These results further indicate the potential usefulness of cfDNA even in patients with low levels of BM infiltration.

\section{CXCR4 genotype assessment in cfDNA and tDNA}

We next examined the feasibility of using cfDNA in detecting mutations in $C X C R 4$ gene. Of the 98 patients with IgM monoclonal gammopathies, mutational screening of CXCR4 gene could be evaluated in 79 patients with cfDNA (79/98 patients, $80 \%)$ and in 73 patients with tDNA (73/98 patients, 74\%). The assessment of CXCR4 mutations in both tDNA and cfDNA was feasible in 65 patients (65 out of 98 patients, 66\%). In eleven patients (11 out of $65,17 \%$ ), mutations were detected in both paired samples. CXCR4 mutations by either cfDNA or tDNA were present in 2 out of 9 MGUS (22\%) patients, 10 out of $37(27 \%)$ patients with disease in remission, and in 6 out of 29 (20\%) patients with sWM/NDWM/RRWM. The pathogenic mutation $S 338 X$ (rs104893626) was present in three patients in one patient with sWM and in two with NDWM. Figure 3 illustrates the sequencing chromatogram of S338X mutation as presented in tDNA and cfDNA. The S338X mutation results from a single nucleotide $\mathrm{C}$ to $\mathrm{G}$ change leading to a predicted stop codon in place of a serine at amino acid position 338. Mutations found in CXCR4 gene are presented in Table 3. Synonymous mutations were found in five patients. Five patients harbored more than one mutation. Fifty-six patients (56 out of 65 patients) were characterized as $C X C R 4^{W T}$ based on both tDNA and cfDNA sequencing. In 3 out of 65 patients, the E343D, $H 228 Q$, and $L 50 X$ truncating mutations were detected in tDNA but not in cfDNA. The L210P mutation (1 out of 65 patients) and the V54G, K110R, and V99G mutations (1 out of 65 patients) were detected in the cfDNA but not in tDNA. Overall, the concordance rate between tDNA and cfDNA was $90 \%$ (59 out of 65 patients). The concordance rate was $100 \%$ among patients with IgM MGUS (7 out of 7 patients), $82 \%$ among WM patients in remission (23 out of 28 patients), and $93 \%$ among patients with sWM/NDWM/ RRWM (28 out of 30 patients). In the four patients with informative tDNA only, one patient presented many polymorphisms in CXCR4 gene, while among eight patients with uninformative tDNA evaluation of cfDNA revealed the $V 99 G$ mutation (1 out of 65 patients) and V82G (1 out of 65 patients). All but S338X (3/65 patients) and V99G (2/65 patients) polymorphisms were presented in one patient. Table 4 illustrates the information regarding our reported polymorphisms in CXCR4 gene. Of these mutations, only S338X and E343D are located in the C-terminal portion, while the rest affect other portions of the protein. As the C-terminal portion plays a critical role in the regulation of signal transduction and CXCR4 expression [31], further analysis is under investigation to determine whether these mutations are tumor specific and clinically relevant to WM pathogenesis. Further analysis will also be conducted for the rest of the detected mutations, especially those which seem to have a higher functional impact according to Provean data (Table 4).

A number of samples with sufficient cfDNA were also tested with primers targeting smaller gene amplicons. This was due to the fact that cfDNA size is relatively small and potentially degraded, and hence may not able to support larger amplicon size. The smaller amplicon targeting primers were tested in 4 samples which had sufficient cfDNA and in which sequencing had been unsuccessful, and in 10 samples which had been successfully sequenced (5 cfDNA and 5 tDNA) in order to test concordance with our first set of primers targeting larger amplicons. We found that samples in which sequencing was not successful with the first set of primers, did not also work with the primers targeting small amplicons. Sequencing with the small amplicon targeting primers gave the same results with samples amplified with the first set of primers.

Regarding the control group of non-IgM MGUS and MM patients, no $C X C R 4$ mutations were detected in tDNA (8 out of 8 patients for $C X C R 4$ ) and cfDNA (7 out of 7 patients for $C X C R 4)$.

\section{Discussion}

This is the first study to evaluate the feasibility of detecting somatic mutations of MYD88 and CXCR4 in the cfDNA derived from the PB plasma of patients with IgM monoclonal gammopathies. The evaluation of cfDNA for mutational characterization and monitoring of disease burden in other hematologic malignancies such as MM has been recently described and shown that cfDNA levels are significantly higher in MM patients compared to other type of cancers [27, 28, 32]. In addition it has been shown that the sensitivity of detecting mutations in plasma was significantly higher compared to the detection in the BM [32]. Although BM examination is required for the diagnosis of 
Fig. 3 Sanger sequencing of cfDNA and tDNA for mutations in CXCR4. WM5 is characterized by the pathogenic S338X mutation as displayed in both tDNA and cfDNA by the sequencing chromatogram
WM5 cfDNA

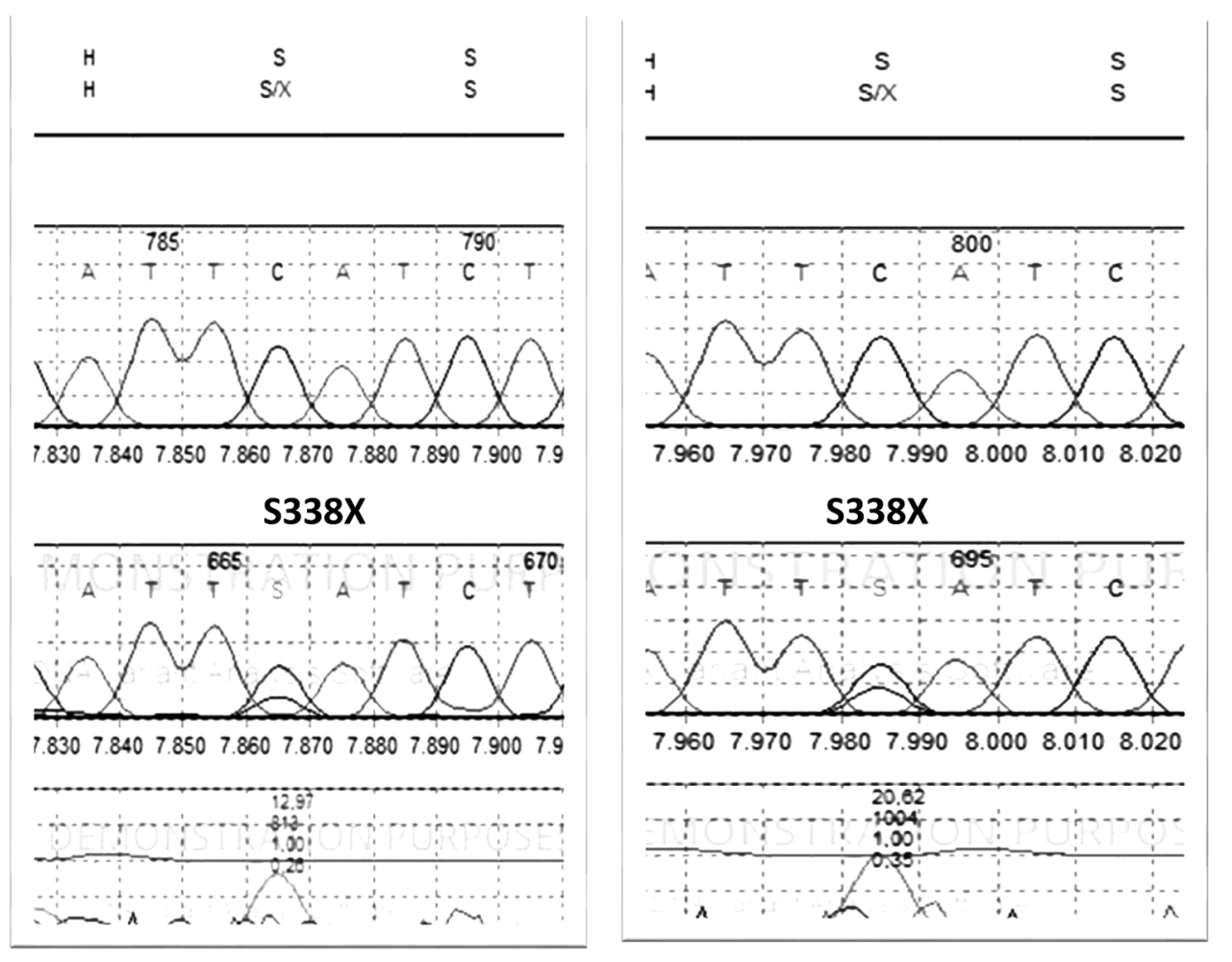

WM, the application of cfDNA could help in monitoring the response to therapy as well as MRD, avoiding the repeated BM sampling. Further studies with larger cohort of WM patients will be performed, to evaluate if this is also the case for detecting mutations in the plasma of these patients.

In our study, we found that in patients with IgM monoclonal gammopathies the MYD88 ${ }^{\mathrm{L} 265 \mathrm{P}}$ mutation and mutations in CXCR4 gene in cfDNA can be detected with a high concordance of 94 and $90 \%$, respectively, compared to those detected in the tDNA. These high concordance rates indicate that cfDNA testing provides are liable diagnostic tool which could replace BM testing in many cases. Table 4 illustrates the rates of detection of $M Y D 88^{\mathrm{L} 265 \mathrm{P}}$ and of mutations in CXCR4 in $\mathrm{CD}_{19}{ }^{+}$-selected cells and in cfDNA according to different disease states. Additional studies are ongoing, to further determine whether these results may improve with further optimizations and more sensitive approaches. In addition collection of serial samples from the same patient before, during, and after treatment is ongoing, which would provide significant information regarding the use of cfDNA as a tool for molecular monitoring, and determine whether in patients with MYD88 ${ }^{\mathrm{L} 265 \mathrm{P}}$ could be used for sensitive detection of MRD after obtaining a deep response.

The prevalence of MYD88 ${ }^{\mathrm{L} 265 \mathrm{P}}(82 \%)$ in our cohort is comparable to the rates observed in other studies [5, 29, 33, 34]. The prevalence rates are also comparable to those achieved using CD19-selected cells from PB [20, 21]. Although previous studies using unselected PB cells from untreated WM patients have shown that the MYD88 L265P mutation can be detected, the detection sensitivity was so low $(39.5 \%)$ that they were prompted to examine the mutation in CD19-selected PB cells using magnetic bead isolation for AS-PCR [20]. In regard to the detection sensitivity of MYD88 L265P mutation, it has been previously reported that although lower BM burden ( $<10 \%$ infiltrate) in patients could result to false negative results, AS-PCR is sensitive enough to detect the L265P mutation in tumor cells as low as $1.25 \%$ [35]. Our results confirm these findings, since in patients with BM infiltrate as low as 5\%, the L265P mutation could be detected in PB cfDNA by both AS-PCR and direct sequencing.

This minimally invasive method could be considered as a cost-effective approach, avoiding the discomfort and risks associated with BM aspiration and the time-consuming tDNA acquirement through CD19 processing (from BM or PB). Most importantly, cfDNA seems to be representative of both the extramedullary disease and the whole BM compartment and not just of a specific site of sampling which might inadequately reflect clonal heterogeneity and inconsistent distribution of clonal cells within the BM, which is typically the case during a BM aspiration or biopsy. The application of cfDNA testing demonstrates the potential for a minimally invasive platform with an 
Table 3 CXCR4 mutation predictions
Table 4 Rates of detection of $M Y D 88^{\mathrm{L} 265 \mathrm{P}}$ and of mutations in CXCR4 in CD19 ${ }^{+}$-selected cells and in cfDNA, according to different disease states in 98 patients with monoclonal IgM

\begin{tabular}{|c|c|c|c|c|c|c|}
\hline \multirow{2}{*}{$\frac{\text { Mutation }(\mathrm{Hg} 19)}{\mathrm{P} 27 \mathrm{~T}}$} & \multicolumn{2}{|c|}{ Mutation (Hg38) } & \multirow{2}{*}{$\begin{array}{l}\text { Gene } \\
\text { CXCR4 }\end{array}$} & \multirow{2}{*}{$\frac{\text { Provean number }}{-2.986}$} & \multirow{2}{*}{$\frac{\text { Prediction }}{\text { Deleterious }}$} & \multirow{2}{*}{$\frac{\text { Reported SNP }}{\text { Not reported }}$} \\
\hline & P31T & & & & & \\
\hline F29L & F33L & & CXCR4 & -0.778 & Neutral & Not reported \\
\hline L50X & L54X & & CXCR4 & & Pathogenic & Not reported \\
\hline I53L & I57L & & CXCR4 & -0.121 & Neutral & rs56400844 \\
\hline V54G & V58G & & CXCR4 & -4.11 & Deleterious & Not reported \\
\hline V82G & V86G & & CXCR4 & -5.5 & Deleterious & Not reported \\
\hline V99G & V103G & & CXCR4 & -1.952 & Neutral & Not reported \\
\hline K110R & K114R & & CXCR4 & -2.4 & Neutral & Not reported \\
\hline L136Q & L140Q & & CXCR4 & -5.5 & Deleterious & Not reported \\
\hline H228Q & H232Q & & CXCR4 & -0.306 & Neutral & rs762625278 \\
\hline S338X & S342X & & CXCR4 & & Pathogenic & rs 104893626 \\
\hline \multirow[t]{2}{*}{ E343D } & E347D & & CXCR4 & -1.244 & Neutral & Not reported \\
\hline & & \multicolumn{2}{|c|}{$\begin{array}{l}\text { CD19+ } \\
\text { MYD88L65P }\end{array}$} & $\begin{array}{l}\text { cfDNA } \\
\text { MYD88L265P }\end{array}$ & $\begin{array}{l}\text { CD19+ } \\
\text { CXCR4mut }\end{array}$ & $\begin{array}{l}\text { cfDNA } \\
\text { CXCR4mut }\end{array}$ \\
\hline \multicolumn{2}{|l|}{ IgM MGUS } & \multicolumn{2}{|c|}{$\begin{array}{l}(N=6) \\
100 \%\end{array}$} & $\begin{array}{l}(N=7) \\
86 \%\end{array}$ & $\begin{array}{l}(N=7) \\
29 \%\end{array}$ & $\begin{array}{l}(N=9) \\
22 \%\end{array}$ \\
\hline \multicolumn{2}{|l|}{ sWM } & \multicolumn{2}{|c|}{$\begin{array}{l}(N=21) \\
95 \%\end{array}$} & $\begin{array}{l}(N=22) \\
82 \%\end{array}$ & $\begin{array}{l}(N=17) \\
29 \%\end{array}$ & $\begin{array}{l}(N=16) \\
19 \%\end{array}$ \\
\hline \multicolumn{2}{|l|}{ NDsWM } & \multicolumn{2}{|c|}{$\begin{array}{l}(N=11) \\
91 \%\end{array}$} & $\begin{array}{l}(N=11) \\
73 \%\end{array}$ & $\begin{array}{l}(N=8) \\
38 \%\end{array}$ & $\begin{array}{l}(N=12) \\
25 \%\end{array}$ \\
\hline \multicolumn{2}{|l|}{$\mathrm{WM}$ in relapse } & \multicolumn{2}{|c|}{$\begin{array}{l}(N=9) \\
100 \%\end{array}$} & $\begin{array}{l}(N=9) \\
100 \%\end{array}$ & $\begin{array}{l}(N=8) \\
0 \%\end{array}$ & $\begin{array}{l}(N=9) \\
0 \%\end{array}$ \\
\hline \multicolumn{2}{|c|}{ WM during active therapy } & \multicolumn{2}{|c|}{$\begin{array}{l}(N=13) \\
69 \%\end{array}$} & $\begin{array}{l}(N=13) \\
62 \%\end{array}$ & $\begin{array}{l}(N=11) \\
27 \%\end{array}$ & $\begin{array}{l}(N=15) \\
27 \%\end{array}$ \\
\hline \multicolumn{2}{|l|}{ WM post therapy } & \multicolumn{2}{|c|}{$\begin{array}{l}(N=26) \\
81 \%\end{array}$} & $\begin{array}{l}(N=24) \\
83 \%\end{array}$ & $\begin{array}{l}(N=22) \\
18 \%\end{array}$ & $\begin{array}{l}(N=20) \\
10 \%\end{array}$ \\
\hline \multicolumn{2}{|l|}{ Overall } & \multicolumn{2}{|c|}{$\begin{array}{l}(N=86) \\
87 \%\end{array}$} & $\begin{array}{l}(N=86) \\
80 \%\end{array}$ & $\begin{array}{l}(N=73) \\
23 \%\end{array}$ & $\begin{array}{l}(N=81) \\
17 \%\end{array}$ \\
\hline
\end{tabular}

increasing range of application in stratifying patients according to their genetic identity. Thus, PB cfDNA could become a useful tool for the initial assessment of $M Y D 88^{L 265 P}$ and CXCR4 mutations by reflecting the overall tumor burden in patients with WM. Furthermore, cfDNA could prevent a repeat BM examination in patients diagnosed with $\operatorname{IgM}$ monoclonal gammopathies and unknown mutational status by minimizing the conventional BMderived tDNA mutation detection to those patients with indeterminate results. To confirm our hypothesis, a larger cohort of patients is tested, in order to obtain a more comprehensive genetic profiling of cfDNA and tDNA in serial samples and assess tumor dynamics by means of cfDNA.

In conclusion, the use of PB cfDNA provides a minimally invasive, but informative and sensitive tool for the assessment of mutational status of MYD88 and CXCR4 of patients with IgM monoclonal gammopathies. Importantly, cfDNA results show high concordance with the results obtained by assessment of BM-derived CD19-positive cells and can be used for the assessment of patients with IgM monoclonal gammopathies.

\section{Key Points}

- $\quad$ MYD88 and CXCR4 mutations were detected in cfDNA of patients with IgM monoclonal gammopathies in high concordance to bone marrow tumor DNA.

- cfDNA is a minimally invasive method for assessing the MYD88 and CXCR4 mutational status that may reduce the need for bone marrow aspiration.

Acknowledgements The authors would like to thank the patients who provided samples for their support of these studies.

Author contributions TB designed research, performed the experiments, and wrote the manuscript. INS analyzed data and wrote the manuscript. MG collected and analyzed patient data. NMK performed the experiments. CL performed the experiments. DP performed the 
experiments. NK collected and analyzed patient samples and data. MM collected and analyzed patient samples and data. DCZ collected and analyzed patient samples and data. EEP collected and analyzed patient samples and data. MR collected and analyzed patient samples and data. DF collected and analyzed patient samples and data. ET wrote the manuscript. EK designed research and wrote the manuscript. MAD wrote the manuscript. All authors critically reviewed, provided input, and approved the final version of the manuscript.

\section{Compliance with ethical standards}

Conflict of interest The authors declare that they have no conflict of interest.

Open Access This article is licensed under a Creative Commons Attribution 4.0 International License, which permits use, sharing, adaptation, distribution and reproduction in any medium or format, as long as you give appropriate credit to the original author(s) and the source, provide a link to the Creative Commons license, and indicate if changes were made. The images or other third party material in this article are included in the article's Creative Commons license, unless indicated otherwise in a credit line to the material. If material is not included in the article's Creative Commons license and your intended use is not permitted by statutory regulation or exceeds the permitted use, you will need to obtain permission directly from the copyright holder. To view a copy of this license, visit http://creativecommons. org/licenses/by/4.0/.

\section{References}

1. Treon SP, Xu L, Yang G, Zhou Y, Liu X, Cao Y, et al. MYD88 L265P somatic mutation in Waldenstrom's macroglobulinemia. $\mathrm{N}$ Eng J Med. 2012;367:826-33.

2. Hunter ZR, Xu L, Yang G, Zhou Y, Liu X, Cao Y, et al. The genomic landscape of Waldenstrom macroglobulinemia is characterized by highly recurring MYD88 and WHIM-like CXCR4 mutations, and small somatic deletions associated with B-cell lymphomagenesis. Blood. 2014;123:1637-46.

3. Treon SP, Cao Y, Xu L, Yang G, Liu X, Hunter ZR. Somatic mutations in MYD88 and CXCR4 are determinants of clinical presentation and overall survival in Waldenstrom macroglobulinemia. Blood. 2014;123:2791-6.

4. Burnworth B, Wang Z, Singleton TP, Bennington A, Fritschle W, Bennington R, et al. Clone-specific MYD88 L265P and CXCR4 mutation status can provide clinical utility in suspected Waldenstrom macroglobulinemia/lymphoplasmacytic lymphoma. Leuk Res. 2016;51:41-8.

5. Abeykoon JP, Paludo J, King RL, Ansell SM, Gertz MA, LaPlant $\mathrm{BR}$, et al. MYD88 mutation status does not impact overall survival in Waldenström macroglobulinemia. Am J Hematol. 2018 Feb;93(2):187-194.

6. Yang G, Zhou Y, Liu X, Xu L, Cao Y, Manning RJ, et al. A mutation in MYD88 (L265P) supports the survival of lymphoplasmacytic cells by activation of Bruton tyrosine kinase in Waldenstrom macroglobulinemia. Blood. 2013;122:1222-32.

7. Ngo VN, Young RM, Schmitz R, Jhavar S, Xiao W, Lim KH, et al. Oncogenically active MYD88 mutations in human lymphoma. Nature. 2011;470:115-9.

8. Hunter ZR, Xu L, Yang G, Tsakmaklis N, Vos JM, Liu X, et al. Transcriptome sequencing reveals a profile that corresponds to genomic variants in Waldenstrom macroglobulinemia. Blood. 2016;128:827-38.
9. Treon SP, Tripsas CK, Meid K, Warren D, Varma G, Green R, et al. Ibrutinib in previously treated Waldenstrom's macroglobulinemia. N Eng J Med. 2015;372:1430-40.

10. Treon SP, Xu L, Hunter Z. MYD88 mutations and response to ibrutinib in Waldenstrom's macroglobulinemia. N Eng J Med. 2015;373:584-6.

11. Dimopoulos MA, Trotman J, Tedeschi A, Matous JV, Macdonald $\mathrm{D}$, Tam $\mathrm{C}$, et al. Ibrutinib for patients with rituximab-refractory Waldenstrom's macroglobulinaemia (iNNOVATE): an open-label substudy of an international, multicentre, phase 3 trial. Lancet Oncol. 2017;18:241-50.

12. Xu L, Hunter ZR, Tsakmaklis N, Cao Y, Yang G, Chen J, et al. Clonal architecture of CXCR4 WHIM-like mutations in Waldenstrom macroglobulinaemia. Br J Haematol. 2016;172:735-44.

13. Azab AK, Runnels JM, Pitsillides C, Moreau AS, Azab F, Leleu $\mathrm{X}$, et al. CXCR4 inhibitor AMD3100 disrupts the interaction of multiple myeloma cells with the bone marrow microenvironment and enhances their sensitivity to therapy. Blood. 2009;113: 4341-51.

14. Ngo HT, Leleu X, Lee J, Jia X, Melhem M, Runnels J, et al. SDF1/CXCR4 and VLA-4 interaction regulates homing in Waldenstrom macroglobulinemia. Blood. 2008;112:150-8.

15. Roccaro AM, Sacco A, Shi J, Chiarini M, Perilla-Glen A, Manier $\mathrm{S}$, et al. Exome sequencing reveals recurrent germ line variants in patients with familial Waldenstrom macroglobulinemia. Blood. 2016;127:2598-606.

16. Sacco A, Fenotti A, Affo L, Bazzana S, Russo D, Presta M, et al. The importance of the genomic landscape in Waldenstrom's macroglobulinemia for targeted therapeutical interventions. Oncotarget. 2017;8:35435-44.

17. Cao Y, Hunter ZR, Liu X, Xu L, Yang G, Chen J, et al. The WHIM-like CXCR4(S338X) somatic mutation activates AKT and ERK, and promotes resistance to ibrutinib and other agents used in the treatment of Waldenstrom's macroglobulinemia. Leukemia. 2015;29:169-76

18. Cao Y, Hunter ZR, Liu X, Xu L, Yang G, Chen J, et al. CXCR4 WHIM-like frameshift and nonsense mutations promote ibrutinib resistance but do not supplant MYD88(L265P) -directed survival signalling in Waldenstrom macroglobulinaemia cells. Br J Haematol. 2015;168:701-7.

19. Roccaro AM, Sacco A, Jimenez C, Maiso P, Moschetta M, Mishima Y, et al. C1013G/CXCR4 acts as a driver mutation of tumor progression and modulator of drug resistance in lymphoplasmacytic lymphoma. Blood. 2014;123:4120-31.

20. Xu L, Hunter ZR, Yang G, Cao Y, Liu X, Manning R, et al. Detection of MYD88 L265P in peripheral blood of patients with Waldenstrom's macroglobulinemia and IgM monoclonal gammopathy of undetermined significance. Leukemia. 2014;28: 1698-704.

21. Growkova K, Kufova Z, Sevcikova T, Filipova J, Kascak M, Jelinek $\mathrm{T}$, et al. Diagnostic tools of Waldenstroms macroglobulinemia-best possibilities for non-invasive and long-term disease monitoring. Klin Onkol. 2017;30:81-91.

22. Siravegna G, Marsoni S, Siena S, Bardelli A. Integrating liquid biopsies into the management of cancer. Nat Rev Clin Oncol. 2017;14:531-48.

23. Diaz LA Jr, Bardelli A. Liquid biopsies: genotyping circulating tumor DNA. J Clin Oncol. 2014;32:579-86.

24. Armand P, Oki Y, Neuberg DS, Faham M, Cummings C, Klinger $\mathrm{M}$, et al. Detection of circulating tumour DNA in patients with aggressive B-cell non-Hodgkin lymphoma. $\mathrm{Br} \mathrm{J}$ Haematol. 2013;163:123-6.

25. Roschewski M, Dunleavy K, Pittaluga S, Moorhead M, Pepin F, Kong K, et al. Circulating tumour DNA and CT monitoring in patients with untreated diffuse large B-cell lymphoma: a correlative biomarker study. Lancet Oncol. 2015;16:541-9. 
26. Yeh P, Hunter T, Sinha D, Ftouni S, Wallach E, Jiang D, et al. Circulating tumour DNA reflects treatment response and clonal evolution in chronic lymphocytic leukaemia. Nat Commun. 2017;8:14756.

27. Oberle A, Brandt A, Voigtlaender M, Thiele B, Radloff J, Schulenkorf A, et al. Monitoring multiple myeloma by nextgeneration sequencing of $\mathrm{V}(\mathrm{D}) \mathrm{J}$ rearrangements from circulating myeloma cells and cell-free myeloma DNA. Haematologica. 2017;102:1105-11.

28. Rustad EH, Coward E, Skytoen ER, Misund K, Holien T, Standal T, et al. Monitoring multiple myeloma by quantification of recurrent mutations in serum. Haematologica. 2017;102:1266-72.

29. Xu L, Hunter ZR, Yang G, Zhou Y, Cao Y, Liu X, et al. MYD88 L265P in Waldenstrom macroglobulinemia, immunoglobulin $\mathrm{M}$ monoclonal gammopathy, and other B-cell lymphoproliferative disorders using conventional and quantitative allele-specific polymerase chain reaction. Blood. 2013;121:2051-8.

30. Mori N, Ohwashi M, Yoshinaga K, Mitsuhashi K, Tanaka N, Teramura M, et al. L265P mutation of the MYD88 gene is frequent in Waldenstrom's macroglobulinemia and its absence in myeloma. PLoS ONE. 2013;8:e80088.
31. Poulain S, Roumier C, Venet-Caillault A, Figeac M, Herbaux C, Marot G, et al. Genomic landscape of CXCR4 mutations in Waldenstrom macroglobulinemia. Clin Cancer Res. 2016;22: 1480-8.

32. Mithraprabhu S, Spencer A. Circulating tumour DNA analysis in multiple myeloma. Oncotarget. 2017;8:90610-1.

33. Gachard N, Parrens M, Soubeyran I, Petit B, Marfak A, Rizzo D, et al. IGHV gene features and MYD88 L265P mutation separate the three marginal zone lymphoma entities and Waldenstrom macroglobulinemia/lymphoplasmacytic lymphomas. Leukemia. 2013;27:183-9.

34. Varettoni M, Arcaini L, Zibellini S, Boveri E, Rattotti S, Riboni $\mathrm{R}$, et al. Prevalence and clinical significance of the MYD88 (L265P) somatic mutation in Waldenstrom's macroglobulinemia and related lymphoid neoplasms. Blood. 2013;121:2522-8.

35. Staiger AM, Ott MM, Parmentier S, Rosenwald A, Ott G, Horn H, et al. Allele-specific PCR is a powerful tool for the detection of the MYD88 L265P mutation in diffuse large B cell lymphoma and decalcified bone marrow samples. Br J Haematol. 2015;171: $145-8$. 